\title{
In vivo studies of cartilage regeneration after damage induced by catabolin/interleukin-1
}

\author{
D P Page Thomas, B King, T Stephens, J T Dingle
}

\begin{abstract}
The response of the rabbit knee joint to a brief episode of cytokine induced damage is described. After three intra-articular injections of catabolin/interleukin-1 all joint cartilages showed an immediate extensive loss of proteoglycan (glycosaminoglycan), which was gradually replaced over three to four weeks. Glycosaminoglycan biosynthesis (measured by ${ }^{35} \mathrm{SO}_{4}$ uptake) was initially depressed, but at one week had almost doubled its rate as compared with the normal side. This increased synthetic activity was further maintained throughout the duration of the experiment (28 days), though the rate gradually fell. Histological cartilage metachromasia to toluidine blue mirrored the glycosaminoglycan changes. No disturbance of the articular cartilage collagen network was found.

It is considered, therefore, that during treatment for arthritis the indigenous chondrocyte must continue to be capable of carry-
\end{abstract}

ing out regenerative matrix repair and that antiarthritic agents should first be screened for interference with that process.

Substantial loss of cartilage proteoglycan and simultaneous inhibition of glycosaminoglycan (GAG) synthesis has been shown to occur after the intra-articular injection into rabbits of a synovial preparation of catabolin/interleukin-1 (IL-1). ${ }^{1}$ The loss of matrix materials was accompanied by a rise in the GAG concentration in the synovial fluid of the injected joint. The principal action of the partially purified synovial preparation was almost certainly due to IL-1 as Pettipher et al have shown that human recombinant IL-1 caused similar changes when injected into rabbit knee joints. ${ }^{2}$ Experiments with human recombinant IL-1 $\alpha$ in this laboratory have confirmed the findings of Pettipher $e t$ $a l$, but in our hands the purified recombinant material, though of similar activity by in vitro

Table 1 Cartilage matrix glycosaminoglycan changes

\begin{tabular}{|c|c|c|c|c|c|c|}
\hline \multirow[t]{2}{*}{ Cartilage } & \multicolumn{6}{|c|}{ Days after injection } \\
\hline & 1 & 3 & 7 & 14 & 21 & 28 \\
\hline $\begin{array}{l}\text { Patella } \\
\text { Normal (N) (mean) } \\
\text { SEM } \\
\text { Treated (Tr) (mean) } \\
\text { SEM } \\
(\text { Tr-N) } \\
\text { SEM } \\
\text { p Value } \\
(\operatorname{Tr} / \mathbf{N}) \times 100\end{array}$ & $\begin{array}{c}849 \\
62 \\
309 \\
25 \\
-540 \\
46 \\
<0.001 \\
36\end{array}$ & $\begin{array}{c}806 \\
86 \\
429 \\
45 \\
-377 \\
72 \\
<0 \cdot 01 \\
53\end{array}$ & $\begin{array}{c}820 \\
94 \\
404 \\
56 \\
-416 \\
61 \\
<0 \cdot 01 \\
49\end{array}$ & $\begin{array}{c}689 \\
51 \\
512 \\
24 \\
-177 \\
30 \\
<0.01 \\
74\end{array}$ & $\begin{array}{c}734 \\
41 \\
604 \\
52 \\
-130 \\
46 \\
<0 \cdot 05 \\
82\end{array}$ & $\begin{array}{c}842 \\
109 \\
794 \\
132 \\
-48 \\
44 \\
\mathrm{NS} \\
94\end{array}$ \\
\hline $\begin{array}{l}\text { Femur } \\
\text { Normal (mean) } \\
\text { SEM } \\
\text { Treated (mean) } \\
\text { SEM } \\
(\mathrm{Tr}-\mathrm{N}) \\
\text { SEM } \\
\text { p Value } \\
(\mathrm{Tr} / \mathrm{N}) \times 100\end{array}$ & $\begin{array}{c}2901 \\
200 \\
1192 \\
77 \\
-1709 \\
161 \\
<0 \cdot 001 \\
41\end{array}$ & $\begin{array}{c}3148 \\
116 \\
1647 \\
45 \\
-1501 \\
104 \\
<0.001 \\
52\end{array}$ & $\begin{array}{c}3066 \\
401 \\
1592 \\
229 \\
-1474 \\
238 \\
<0.01 \\
52\end{array}$ & $\begin{array}{c}2752 \\
354 \\
2690 \\
266 \\
-62 \\
30 \\
<0.01 \\
98\end{array}$ & $\begin{array}{r}3235 \\
124 \\
3109 \\
209 \\
-126 \\
135 \\
\text { NS } \\
96\end{array}$ & $\begin{array}{c}2868 \\
212 \\
3269 \\
250 \\
401 \\
112 \\
<0 \cdot 02 \\
114\end{array}$ \\
\hline $\begin{array}{l}\text { Tibia } \\
\text { Normal (mean) } \\
\text { SEM } \\
\text { Treated (mean) } \\
\text { SEM } \\
(\mathrm{Tr}-\mathrm{N}) \\
\text { SEM } \\
\text { p Value } \\
(\operatorname{Tr} / \mathbf{N}) \times 100\end{array}$ & $\begin{array}{c}2244 \\
144 \\
1107 \\
239 \\
-1137 \\
146 \\
<0 \cdot 001 \\
49\end{array}$ & $\begin{array}{c}3072 \\
220 \\
1700 \\
170 \\
-1372 \\
103 \\
<0 \cdot 001 \\
55\end{array}$ & $\begin{array}{c}2697 \\
218 \\
1415 \\
116 \\
-1282 \\
172 \\
<0 \cdot 001 \\
52\end{array}$ & $\begin{array}{r}2312 \\
99 \\
2252 \\
299 \\
-60 \\
221 \\
\text { NS } \\
97\end{array}$ & $\begin{array}{c}3044 \\
52 \\
2749 \\
41 \\
-295 \\
68 \\
<0 \cdot 01 \\
90\end{array}$ & $\begin{array}{r}2920 \\
217 \\
2810 \\
238 \\
-110 \\
162 \\
\text { NS } \\
96\end{array}$ \\
\hline $\begin{array}{l}\text { Meniscus } \\
\text { Normal (mean) } \\
\text { SEM } \\
\text { Treated (mean) } \\
\text { SEM } \\
(\text { Tr-N) } \\
\text { SEM } \\
\text { p Value } \\
(\operatorname{Tr} / \mathbf{N}) \times 100\end{array}$ & $\begin{array}{c}1029 \\
50 \\
758 \\
36 \\
-271 \\
66 \\
<0.01 \\
74\end{array}$ & $\begin{array}{c}1064 \\
94 \\
760 \\
71 \\
-304 \\
30 \\
<0.001 \\
71\end{array}$ & $\begin{array}{c}1068 \\
86 \\
617 \\
67 \\
-451 \\
37 \\
<0 \cdot 001 \\
58\end{array}$ & $\begin{array}{l}955 \\
110 \\
872 \\
155 \\
-83 \\
76 \\
\text { NS } \\
91\end{array}$ & $\begin{array}{r}935 \\
86 \\
861 \\
110 \\
-74 \\
102 \\
\text { NS } \\
92\end{array}$ & $\begin{array}{c}885 \\
75 \\
1101 \\
125 \\
216 \\
76 \\
<0 \cdot 05 \\
124\end{array}$ \\
\hline
\end{tabular}

Strangeways Research Laboratory,

Worts Causeway, Cambridge CBI 4RN, UK

D P Page Thomas

B King

T Stephens

J T Dingle

Correspondence to:

Dr Page Thomas.

Accepted for publication 14 August 1990
The values in the table are the mean and SEM values ( $\mu$ g glycosaminoglycan per total joint cartilage), their mean differences, and standard errors found in the various cartilages. As the pairs of cartilages give highly correlated values statistical analyses were made
using the paired $t$ test. The value at each time point is the mean for six animals. 
assay, proved less effective in causing cartilage damage in vivo. It seems likely that the synovial preparation contains a number of active agents, including IL-1 $\alpha, \mathrm{IL}-1 \beta$, and tumour necrosis agent. As explained in the previous paper ${ }^{1}$ it was felt worthwhile to use this material because it may more nearly represent the type of stimulus that cartilage chrondrocytes receive from adjacent pathological synovial tissue. The same arguments apply to this study. Preliminary experiments with recombinant material suggest that the cartilage regeneration pattern will follow a similar course to that reported in this paper using synovial catabolin/IL-1.

Figure 1 Normal fabella. Figures 1-7 show sections of the fabella, which articulates with the lateral femoral condyle. All stained with toluidine blue and photographed using an interference filter (Balzer $\lambda$ 564). Changes in metachromasia of the articular cartilage around and in between chondrocytes are shown as differing densities of dark grey. Bars $=0.01 \mathrm{~mm}$.

Figure 2 One day after last injection. Complete loss of metachromasia above ossifying cartilage. See fig 1 caption.

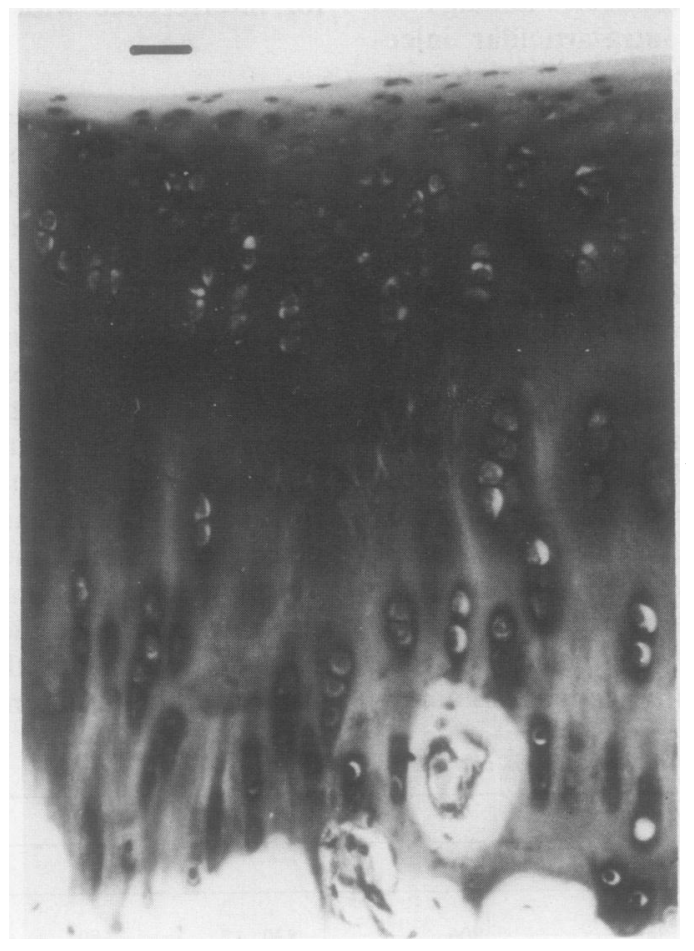
factor as well, perhaps, as uncharacterised

Materials and methods

The methods used for GAG assay and conditions for ${ }^{35} \mathrm{SO}_{4}$ uptake were those previously described. ${ }^{1}$ Briefly, old English rabbits (2-3 kg) were injected into one knee joint (randomly allocated) with the cytokine preparation on three occasions at three day intervals. The preparation was standardised to give a dose the equivalent of $24 \mathrm{ng}$ of human recombinant IL$1 \alpha$ per injection. The experimental groups (six

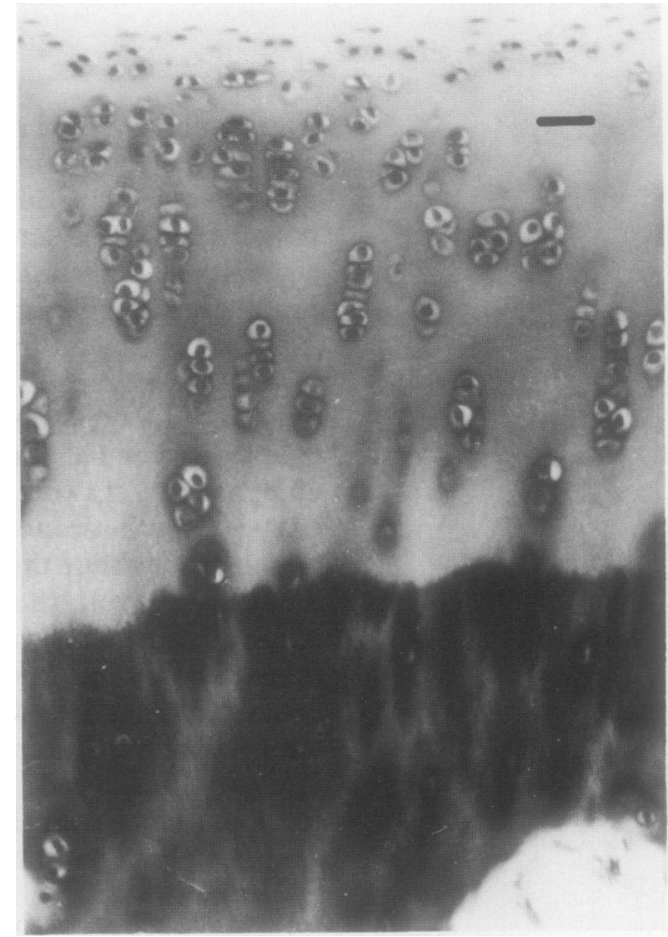

Figure 3 Three days after last injection. Extensive loss of metachromasia persists. See fig 1 caption.

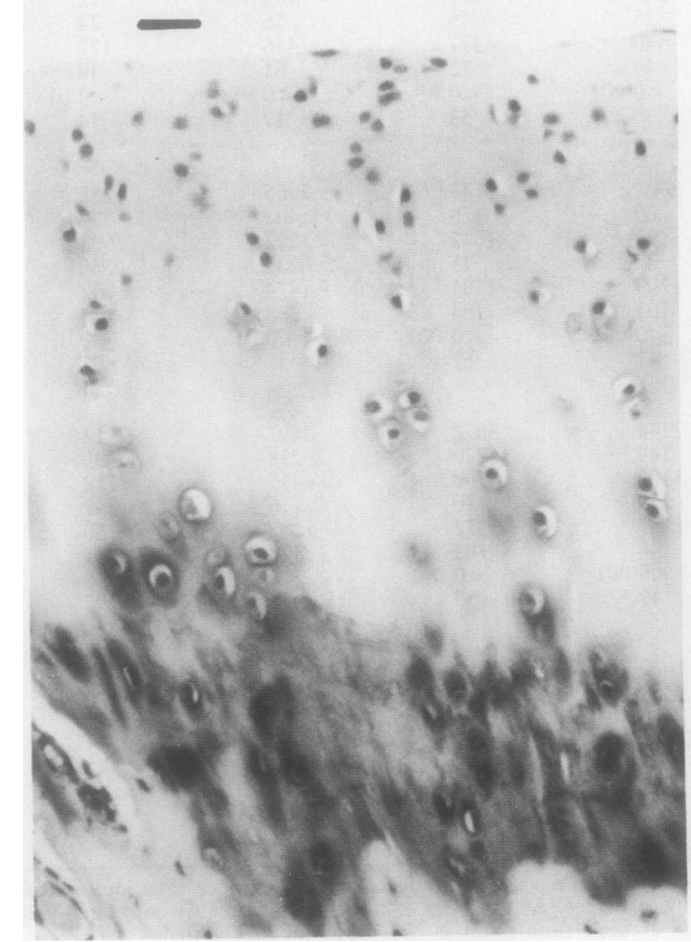

Figure 4 At seven days perichondrocyte metachromasia starting to reappear. The interterritorial regions are still pale. See fig 1 caption. 
animals/group) were terminated at 1, 3, 7, 14, 21 , and 28 days after the last injections. At the end of each experimental period the GAG content of the articular cartilages of the patella, femoral condyles, tibial plateaux, and meniscal fibrocartilages was measured, and after an overnight pulse the ${ }^{35} \mathrm{SO}_{4}$ uptake of the patella and femoral cartilages was estimated. The infrapatellar synovium, the rectus femoris fibrocartilage, and the posterior synovium and fabellae were taken routinely for histology.

\section{Results}

EFFECT UPON GAG CONTENT OF JOINT CARTILAGE Table 1 shows the total GAG content of the articular cartilage of the patella, femoral condyles, tibial plateaux, and meniscal cartilages. The whole joint cartilage values ( $\mu$ g GAG) are the mean of results for six animals, their mean differences, the percentages of the normal sides, and their respective standard errors of the means (SEM). Each animal had one knee injected with catabolin/IL-1 and the other knee was untreated. The animals were killed at intervals between one and 28 days. At one day after injection there was a substantial loss of GAG content in all cartilages, confirming the results of the previous paper. ${ }^{1}$ This significant decrease in GAG content in the various cartilages continued for a further week. At two and three weeks after the cessation of catabolin/IL-1 treatment signs of GAG regeneration were present and significant depression of cartilage values was no longer uniform. Both the femoral cartilage and the meniscal cartilage showed some degree of overshoot in GAG content at 28 days, reflecting the intense synthetic activity of the indigenous chondrocytes.

Table 1 also shows the rate of recovery of the articular cartilage, expressed as a percentage of the treated over the normal knee. This is the

Figure 5 Fourteen days. Metachromasia expanding further. See fig 1 caption. most effective way of comparing the regenerative pattern of the various joint cartilages because the biological variation between animal groups is minimised. It can be seen that the rate of recovery in the femur and the tibia was similar, but in the patella and the meniscus cartilage regeneration was somewhat slower. The meniscal cartilage also showed a somewhat slower rate of loss occurring during treatment, and apparently, the loss continued after cessation of treatment for about one week-a finding not seen in the other articular cartilages. Figures 1-7 show the synchronous histological changes in the fabellae with their normal controls. It is apparent from these representative illustrations that the articular cartilages of these small sesamoid bones fully retained their overall normal architecture throughout, apart from a marked initial loss of toluidine blue metachromasia, which extended down to the 'tide mark' of ossifying chondrocytes. From the first week onwards a progressive increase of intense metachromasia extended outwards from the immediate perilacunar area (seven days), which finally spread over all the interterritorial regions by the third week. These histological findings thus closely mirrored the overall patterns of GAG changes in the biochemical studies. With this particular injection schedule pannus was not found, and the van Gieson staining for collagen remained unaffected.

EFFECT UPON ${ }^{35} \mathrm{SO}_{4}$ UPTAKE

The synthetic activity of the patella and femoral cartilages was measured by incorporation of ${ }^{35} \mathrm{SO}_{4}$ into cetyl pyridinium chloride precipitable material, which is effectively a measurement of GAG synthesis (see table 2). As was expected

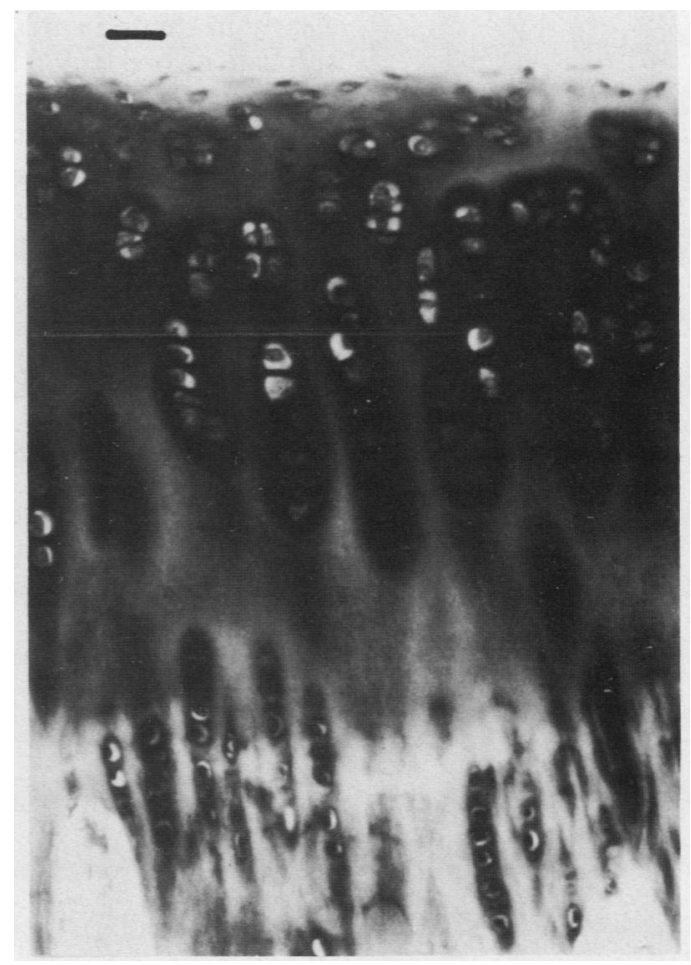

Figure 6 Twenty one days. Metachromasia now fills the interterritorial regions but some uneven density of staining is still present. See fig 1 caption. 


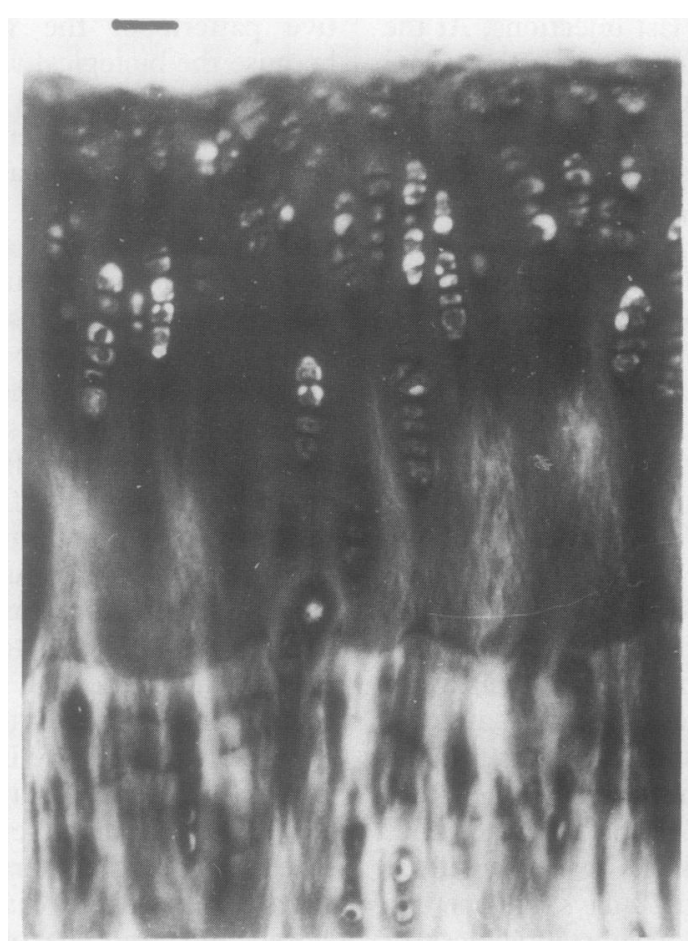

Figure 7 Twenty eight days. Metachromasia has a normal distribution. See fig I caption.

from previous work there was substantial inhibition of GAG synthesis at one day after injection, which continued for a further two days in both cartilages. By one week, however, the patellar and femoral cartilages from the injected knees showed substantially more active synthesis of GAG than was found in normal cartilage. This increased synthetic rate continued for the remainder of the period investigated-that is, up to 28 days. A comparison of the treated and control tissues is seen most clearly in the measurements of the ratio of the sulphate incorporation as the percentage treated over normal, where it was shown that the rate of increase was similar in the two tissues. Although synthetic activity peaked at seven days, there was still substantially more activity in the regenerating cartilage than in the control cartilage during the entire period of the investigation.
HISTOLOGICAL CHANGES IN OTHER JOINT TISSUES Fibrocartilage of rectus femoris

Fibrocartilage of rectus femoris, which runs in the upper part of the intercondylar groove, also took part in the general GAG pattern of changes, showing initial loss and molecular regeneration of the matrix over the next two weeks.

\section{Synovium}

At one day after the last injection there was a viscous effusion which contained large numbers of cells, principally polymorphonuclear leucocytes (fig 8). Subsurface capillaries and postcapillary venules were engorged and often mar-

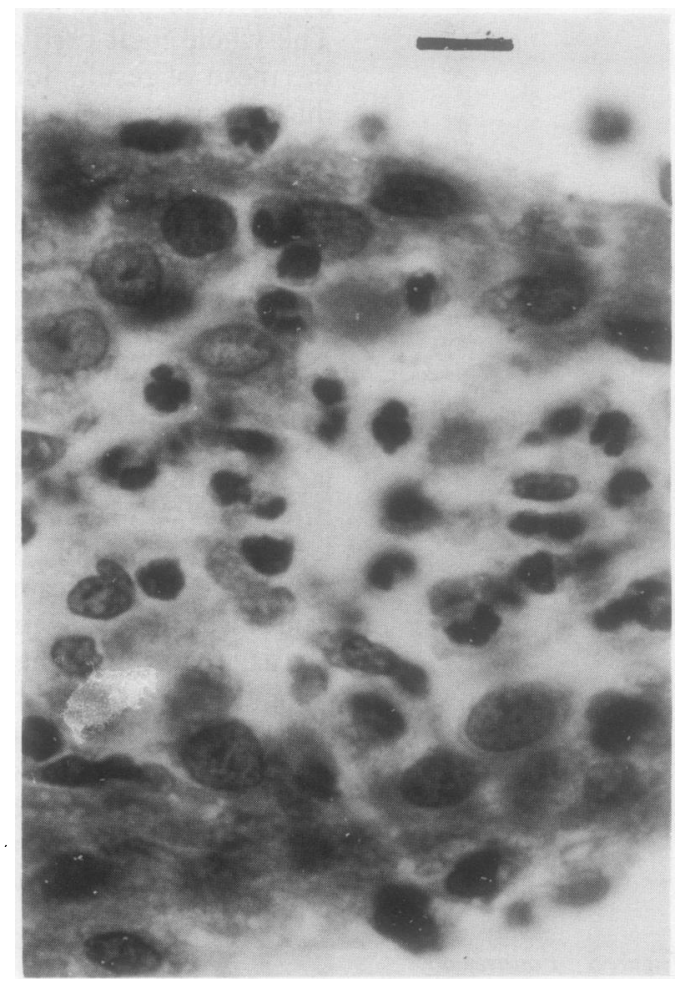

Figure 8 One day synovium. Acute inflammatory changes, a hypertrophic surface layer and migrating polymorphonuclear leucocytes. Figures 8-12 show synchronous synovial changes. All stained with haematoxylin and eosin using an interference filter (Balzer $\lambda$ 564). Bars $=0.01 \mathrm{~mm}$.

Table $2{ }^{35} \mathrm{SO}_{4}$ uptake (glycosaminoglycan synthesis)

\begin{tabular}{|c|c|c|c|c|c|c|}
\hline \multirow[t]{2}{*}{ Cartilage } & \multicolumn{6}{|c|}{ Days after injection } \\
\hline & $l$ & 3 & 7 & 14 & 21 & 28 \\
\hline $\begin{array}{l}\text { Patella } \\
\text { Normal (N) (mean) } \\
\text { SEM } \\
\text { Treated (Tr) (mean) } \\
\text { SEM } \\
(\text { Tr-N) } \\
\text { SEM } \\
\text { p Value } \\
(\text { Tr/N) } \times 100\end{array}$ & $\begin{array}{rl}67 & 392 \\
7 & 069 \\
38 & 689 \\
3 & 244 \\
-28 & 703 \\
5 & 410 \\
& <0 \cdot 01 \\
& 57\end{array}$ & $\begin{array}{rl}70 & 597 \\
7 & 713 \\
47 & 381 \\
11 & 387 \\
-23 & 216 \\
7 & 108 \\
& <0.05 \\
& 67\end{array}$ & $\begin{aligned} 57117 \\
4349 \\
102958 \\
9923 \\
45841 \\
8080 \\
<0.01 \\
180\end{aligned}$ & $\begin{array}{rl}55 & 158 \\
3384 \\
104467 \\
10391 \\
49309 \\
9630 \\
<0.01 \\
189\end{array}$ & $\begin{array}{rl}54 & 917 \\
5 & 021 \\
72 & 880 \\
10 & 485 \\
17 & 963 \\
6 & 347 \\
& <0.05 \\
& 133\end{array}$ & $\begin{array}{rl}67 & 316 \\
9 & 197 \\
81 & 994 \\
8 & 060 \\
14 & 678 \\
5 & 032 \\
& <0 \cdot 05 \\
& 122\end{array}$ \\
\hline $\begin{array}{l}\text { Femur } \\
\text { Normal (mean) } \\
\text { SEM } \\
\text { Treated (mean) } \\
\text { SEM } \\
(\operatorname{Tr}-\mathrm{N}) \\
\text { SEM } \\
\text { p Value } \\
(\mathrm{Tr} / \mathrm{N}) \times 100\end{array}$ & $\begin{array}{rl}175 & 636 \\
8454 \\
101197 \\
5615 \\
-74439 \\
7855 \\
<0.001 \\
58\end{array}$ & $\begin{array}{rl}175 & 280 \\
8 & 973 \\
141 & 305 \\
7 & 645 \\
-33 & 975 \\
11 & 666 \\
& <0.05 \\
& 81\end{array}$ & $\begin{aligned} 151650 \\
5917 \\
287905 \\
13920 \\
136255 \\
19652 \\
<0.001 \\
190\end{aligned}$ & $\begin{aligned} 160436 \\
10081 \\
277900 \\
16406 \\
117464 \\
14990 \\
<0 \cdot 001 \\
173\end{aligned}$ & $\begin{aligned} 159770 \\
15090 \\
220613 \\
27813 \\
60843 \\
18155 \\
<0.05 \\
138\end{aligned}$ & $\begin{array}{rl}142580 \\
8 & 862 \\
173014 \\
5533 \\
30434 \\
4237 \\
<0 \cdot 001 \\
121\end{array}$ \\
\hline
\end{tabular}

The table values (dpm) are the mean counts of the normal and treated knees and their differences per total joint cartilages. Note that the latter values range both negatively (depression of synthesis) and positively (stimulation of synthesis). 


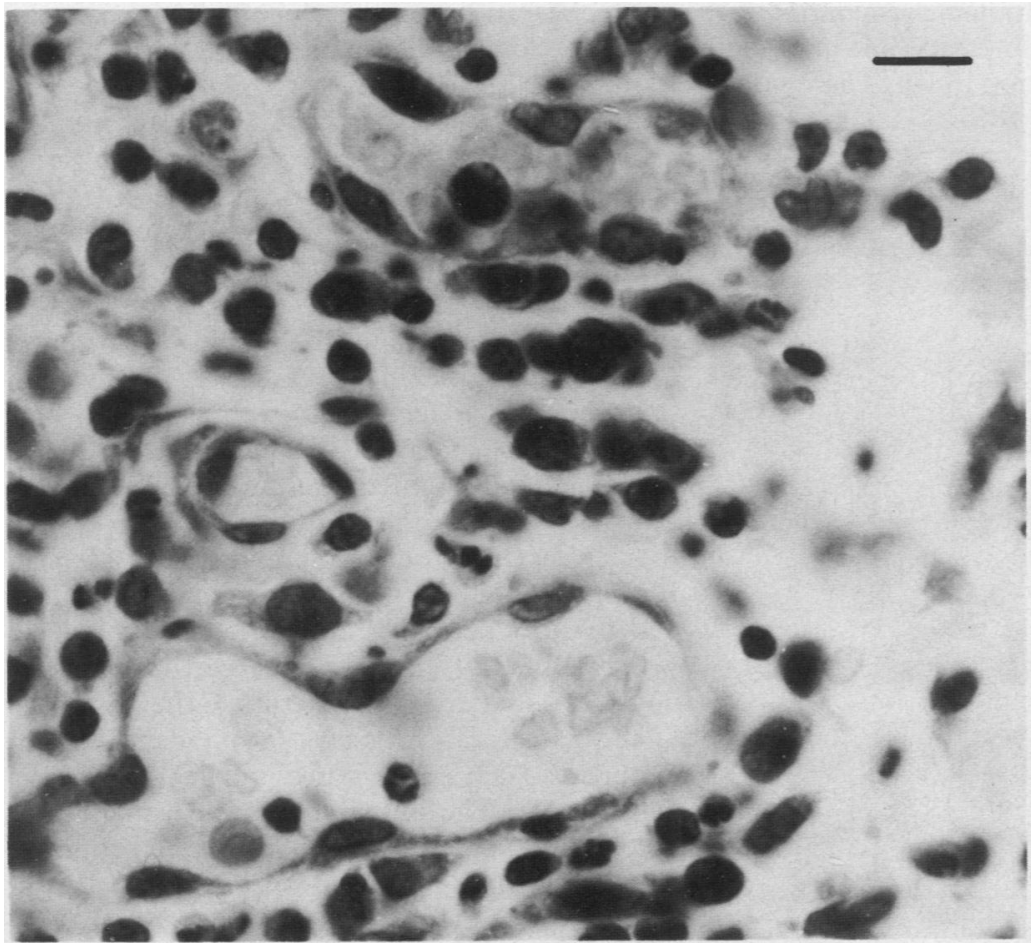

Figure 9 One day. Enlarged endothelial cells in capillaries, and post-capillary venules. See fig 8 caption.

ginating polymorphonuclear leucocytes were present (fig 9). On the third day the effusion was still present, though smaller in amount, but the cellular influx continued. The synovial layer was three to four cells thick, and some subsynovial lymphocyte aggregations were found. Mitosis was present at the surface (fig 10) and in deeper tissues. By seven days the obvious signs of effusion had disappeared and joint washouts contained many fewer leucocytes where polymorphonuclear leucocytes were less in evidence and more macrophages and lymphocytes were found. The lymphocyte aggregations and plasma cell infiltration continued for about three weeks (figs 11 and 12). Finally, at four weeks the appearances were primarily of fibrosis affecting the whole synovium.
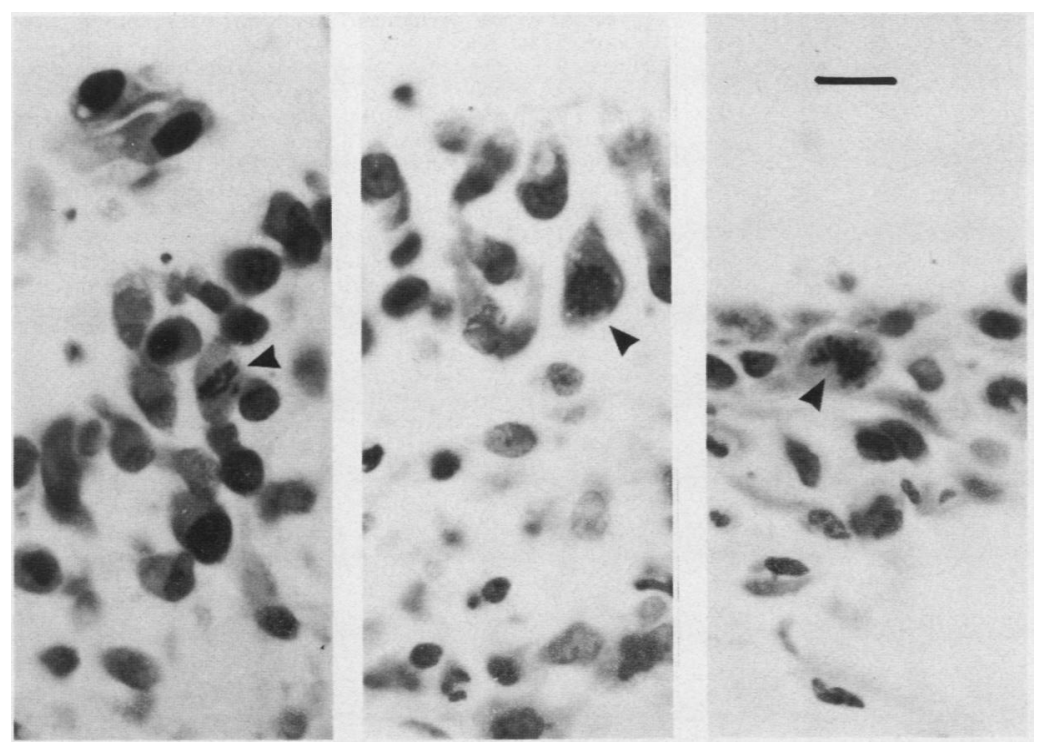

Figure 10 Three days. Mitotic figures throughout synovium. The 'arrow heads' indicate the dividing cells. Three examples from the synovial surface. See fig 8 caption.

\section{Discussion}

As far as we are aware there have been no experiments reported on the regeneration of articular cartilage in vivo after treatment with cytokine preparations. It has been known for some 30 years that matrix regeneration is possible, from the classical experiments of Thomas. $^{3} \mathrm{He}$ showed that the injection of papain into young rabbits caused their ears to droop because of loss of proteoglycan from the ear cartilage, and that, subsequently, the ears returned to an erect position, which corresponded to a return of metachromasia in the cartilage of the ear. In addition, there have been some clinical observations suggesting that recovery can take place after extensive damage in humans. ${ }^{4}$

The experiments characterised in the first paper in this series on the action of synovial catabolin/IL-1 on cartilage integrity showed that the technique was reproducible and quantitative. ${ }^{1}$ It was therefore suitable for the direct estimation of the regeneration of cartilage after damage had been induced in a standard manner. In these experiments we deliberately chose to cause extensive damage-proteoglycan loss of up to $50 \%$ being induced in the matrix. Nevertheless, the chondrocytes retained the ability to regenerate completely the proteoglycan of the matrix. This characteristic was shown both biochemically (by GAG content), histologically (by the metachromatic nature of the matrix), and was reflected in the greatly increased synthetic activity indicated by sulphate incorporation. It must be pointed out that the experiments were not taken to the stage at which substantial loss of the collagen component of the matrix had occurred. In such cases we would expect that proteoglycan would escape into the synovial fluid as long as the remaining chondrocytes remained synthetically active. In the current experiments, however, it is clear that chondrocytes are well able to repack the matrix with proteoglycan if the collagenous framework is intact.

The effect of partially purified synovial catabolin/IL-1 on cartilage synthetic activity was first shown in organ cultures ${ }^{5}$ and investigated in detail using pure pig IL-1 by Tyler ${ }^{6}$ and with human recombinant IL-l $\alpha$ by Benton and Tyler. $^{7}$ They were able to show that the inhibition of synthetic activity on cartilage induced by IL-1 occurred at lower doses than those required to cause cartilage resorption. They showed a direct inhibitory effect on core protein synthesis, though there was no alteration in the rate of intracellular transport and secretion of the completed proteoglycan. Although it is difficult to compare the rates of cartilage loss with the time of inhibition of synthetic activity, they suggest that the action of IL-1 to reduce proteoglycan biosynthesis may begin before the initial depletion of the existing matrix. Like the experiments on porcine tissues, the action of human recombinant IL-1 $\alpha$ on adult human articular cartilage also showed that proteoglycan synthesis is very sensitive to inhibition by the cytokine ${ }^{8}$ but its catabolic activation required a higher dose of IL-1. These results emphasise the importance of sustaining synthetic activity 
Figure 11 Seven days. Round cell aggregation. See fig 8 caption.
Figure 12 Fourteen days. Collection of plasma cells lying just beneath the surface. See fig 8 caption. as well as preventing cytokine induced depletion of matrix if the integrity of articular cartilage is to be maintained during the course of arthritic disease.
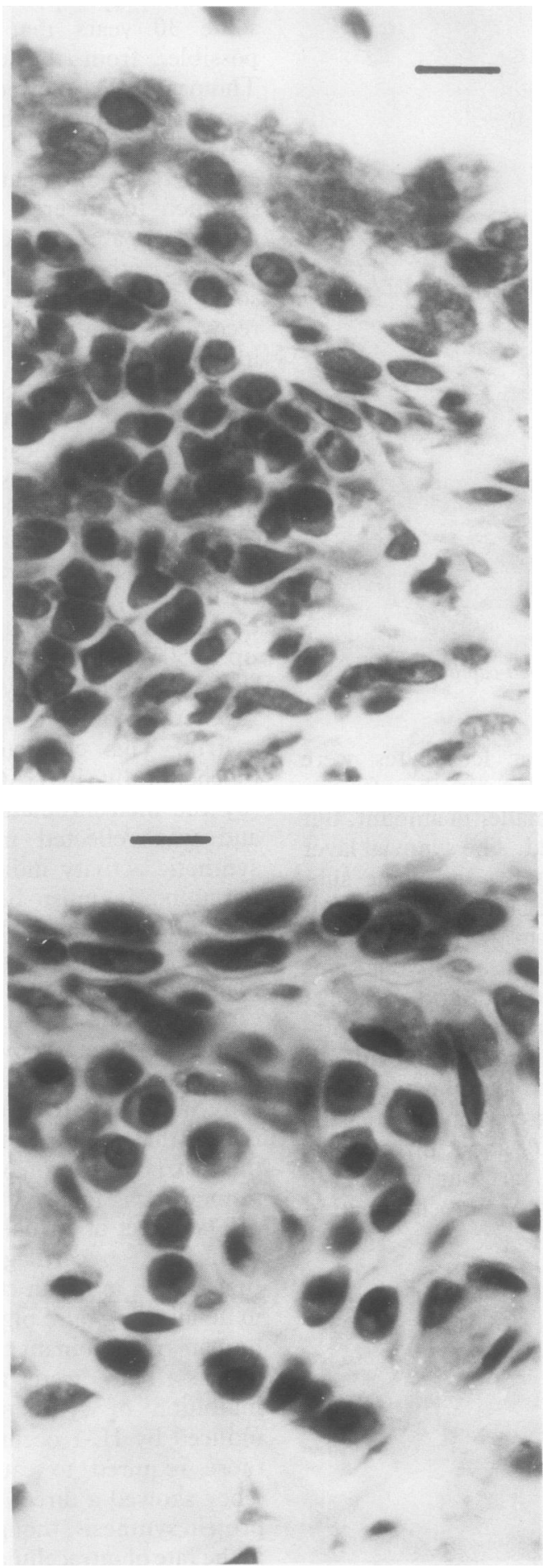

Considerable care must be taken in extrapolating from in vivo experiments on cytokine induced damage in the articular cartilage of rabbits to possible cartilage repair in human arthritic disease, but some general conclusions may be possible. Because articular cartilage is capable of regeneration, even after substantial damage, any treatment designed to modulate inflammation should take into account the importance of maintaining the dynamic balance between synthesis and degradation that occurs in articular cartilage. The possibility that drugs which may be apparently therapeutically useful in diminishing inflammation might simultaneously inhibit the synthetic activity of the indigenous chondrocytes should be considered. If agents could be found to reduce inflammation and the production of cytokines this would be very useful, but only if the chondrocytes were not prevented from regenerating the extracellular matrix. Conversely, any agent which stimulates such regeneration might have therapeutic value. The animal model experiments are potentially useful for investigating the action of agents that may be chondroprotective both by prevention of cytokine production and action, and also by stimulation of the regenerative processes that chondrocytes possess. It will be desirable to know, not only what the commonly employed anti-inflammatory drugs do to the system, but also to determine what action any newly developed agents have on the metabolic activity of human articular cartilage.

DPPT is a member of the external staff of the Medical Research Council. This work was carried out with the aid of funding from the Ciba Geigy Corporation, Summit, New Jersey, USA.

1 Dingle J T, Page Thomas D P, King B, Bard D R. In vivo studies of articular tissue damage mediated by catabolin interleukin-1. Ann Rheum Dis 1987; 46: 527-33.

2 Pettipher E R, Higgs G A, Henderson B. Interleukin 1 induces leucocyte infiltration and cartilage proteoglycan induces leucocyte infiltration and cartilage proteoglycan degradation in the

3 Thomas L J. Reversible collapse of rabbit ears after intravenous papain and prevention of recovery by cortisone. f Exp Med 1956; 104: 245-55.

4 Dieppe P. Osteoarthritis: the scale and scope of the clinical problem. In: Osteoarthritis. current research and prospects for pharmacological intervention. London: IBC Technical Services, 1988: 1-6.

5 Dingle J T. The effect of synovial catabolin on cartilage synthetic activity. Connect Tissue Res 1984; 12: 277-86.

6 Tyler J A. Articular cartilage cultured with catabolin (pig interleukin 1) synthesizes a decreased number of norm proteoglycan molecules. Biochem $\mathcal{J}$ 1985; 227: 869-78.

7 Benton $\mathrm{H}$, Tyler J A. Inhibition of cartilage proteoglycan synthesis by interleukin 1. Biochem Biophys Res Commun 1988; 154: 421-8.

8 Dingle J T. Experimental studies on cartilage degradation and repair. In: Osteoarthritis: current research and prospects and repair. In: Osteoarthritis: current research and prospects Services, 1988: 107-14. 